\title{
DSM-Based Collaborative Flow Optimization Method for Equipment Requirement Demonstration
}

\author{
Y. P. Fan \\ Equipment Command and Management Department \\ Academy of Armored Force Engineering \\ Beijing, China
}

\author{
Q.S. Guo \\ Equipment Command and Management Department \\ Academy of Armored Force Engineering \\ Beijing, China
}

\begin{abstract}
The equipment requirement demonstration is a multidomain, multidisciplinary, multi-department collaborative process. The alternating relationship among the activities are quite complex, which reduces the efficiency of the participant department. So the new method based on design structure matrix is proposed to improve the collaborative relationship among the process of equipment requirement demonstration. The design structure matrix on the flow of equipment requirement demonstration is defined, and the optimizing process and method based on design structure matrix is analyzed in detail. Finally, an example about the flow of equipment type requirement demonstration is discussed. The result shows that the proposed method based on design structure matrix is practical and effective.
\end{abstract}

Keywords-weapon equipment; requirement demonstration; collaborative optimization; design structure matrix

\section{INTRODUCTION}

Equipment requirement demonstration(ERD) is a multidomain, multidisciplinary, multi-department collaborative process. There are large numbers of activities and interaction relations in the process of the ERD[1]. With the increase of the complexity of the equipment system, it's difficult to break down the demonstration mission scientifically, deploy the demonstration resources effectively and improve the efficiency and quality of the ERD. It's the most outstanding characteristic that many departments have to work synchronously in the process of ERD. In other words, a self-governed department can accomplish the work successfully formerly, but nowadays we do it successfully depending on the collaborative work among many participant departments. Thus, the preponderant resources including interrelated software and platforms will be organized better and the collaborative work will be added greatly. However, with the increase of interaction relations among the demonstration activities, the complexity of the process of ERD will increase in a geometric series. As a result, the work efficiency and the quality will decline synchronously. So the collaborative interaction relations among the activities of the process of ERD should be set in a proper number. If the number is larger, the process of ERD will become more complex and the researchers will lose their head in dealing with the work. Contrarily, if the number is fewer, the collaborated goals will not be accomplished well. In order to resolve this problem, a new method based on design structure matrix(DSM) is proposed in this paper. With this method, the number of the collaborative interaction relations among the activities of the process of ERD will be set a proper level, and the process of ERD will be optimized better.

\section{DESCRIPTION OF THE PROBLEM}

The essential requirement developing equipment is the operation demands in the future. According to the operation demands in the future, some representative parameters are proposed to describe the function of the equipment[2]. Generally, the basic contents of ERD are as figured in fig. 1, including operation conception design, operation task requirement analysis, operation capabilities requirement analysis, equipment system requirement analysis, requirement validation and assessment.

The correlative fields are operation, capability and system in the contents. And the basic relationship types among the contents are direction, mapping and feedback. Through interacting among the contents, the expected parameters describing the equipment will be proposed scientifically[3]. However, the collaborative relations among the above contents are not detailed, which cannot lead to a better collaborative work. So the contents have to be divided into detailed actions, and every action had better be required to accomplish individual goal. Thus, the detailed contents and methods of collaboration among various contents will be defined better.

The process of ERD is defined as $D P=\{A, R\}$. The parameter of $A$ is a set of actions, defined as $A=\left\{A_{1}, A_{2}, \cdots, A_{m}\right\}$. The parameter of $R$ is a set of relations among actions, defined as $R=\left\{A_{i}, A_{j}, r_{k}\right\}(i=j=1,2, \cdots, m ; k=1,2,3) \quad$. The

parameters of $r_{1}, r_{2}$ and $r_{3}$ denote the three different relations, such as series-wound relationship, shunt-wound relationship and reactive relationship. 


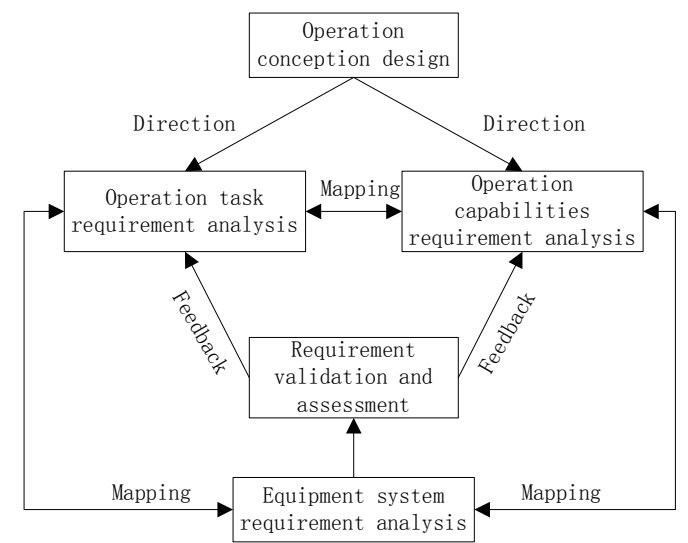

FIGURE I. THE CONTENTS ERD AND THEIR MUTUAL RELATIONSHIP

\section{COLLABORATIVE FLOW OPTIMIZATION METHOD BASED ON DSM FOR ERD}

\section{A. DSM definition}

The method of Design Structure Matrix describes the mutual relationship among the parts of complex system in a matrix. Through some matrix operation such as switching among lines or rows, the interactive relationship among the parts can be reduced greatly. As a result, the complexity of the interactions among the parts can be declined and the conflict among the actions will also be reduced. Therefore, it is regarded as a better method to optimize the process of ERD.

According to definition of DSM[4], the process of ERD is defined as a matrix, showed as figure 2. In this matrix, the dimension of $m$ denotes the number of the actions in the process of ERD. The $m$ actions are defined as $A_{1}, A_{2}, \ldots, A_{m}$. The elements in the diagonal denote the $\mathrm{m}$ actions, and the other elements denote the relationship among the $\mathrm{m}$ actions. So the element of $a_{i j}$ is defined as the following.

$$
a_{i j}=\left\{\begin{array}{c}
A_{i} \quad i=j \\
0 \quad i \neq j \text { and there is no mutual relationship } \\
1 \quad i \neq j \text { and there is mutual relationship }
\end{array}\right.
$$

If the element lays under the diagonal and the value is 1 , the action in ith row receives information from the action in jth line. If the element lays on the diagonal and the value is 1 , the action in ith row receives information from the action in jth line. For example, $a_{21}=1$ denotes the action $A_{2}$ receives information from the action $A_{1}, a_{13}=1$ denotes the action $A_{1}$ receives information from the action $A_{3}$, and there are interactive relationship between the action $A_{1}$ and $A_{3}$.

\begin{tabular}{|c|c|c|c|c|c|}
\hline & $A_{1}$ & $A_{2}$ & $A_{3}$ & $A_{4}$ & $\ldots$ \\
\hline$A_{1}$ & $A_{1}$ & 0 & 1 & 0 & \\
\hline$A_{2}$ & 1 & $A_{2}$ & 0 & 0 & \\
\hline$A_{3}$ & 0 & 0 & $A_{3}$ & 0 & \\
\hline$A_{4}$ & 1 & 1 & 0 & $A$ & \\
\hline 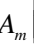 & 1 & 0 & 1 & 1 & \\
\hline
\end{tabular}

FIGURE II. DSM DESCRIBING THE PROCESS OF ERD.

\section{B. Approaches optimizing the flow}

Demonstration flow construction. According to the contents of ERD and their mutual relationships, the actions accomplishing the contents will be proposed and can be organized into a flow directing ERD. Thus, we can clear the structure of the flow and the relationships among the actions, and the executed order of the actions can also be confirmed.

DSM construction. According to the definition of DSM, the DSM describing the process of ERD can be built. The dimension of the matrix is the number of the actions.

DSM reconstruction. With the switch principle, the DSM can be optimized to keep fewer element denoted as 1 lying on the diagonal. After switching the rows or lines repeatedly, the DSM will be closer to the optimization goal.

Demonstration flow reconstruction. According to the optimized DSM, the new flow of ERD can be rebuilt. In this new flow, the reactive relationships among the actions will be simpler than the initial flow.

\section{Method reconstructing DSM}

The reactive relationships among the actions affect the parallel processing of ERD. If the reactive relationships become fewer, the efficiency of parallel processing will be higher. Therefore, through switching the lines and rows of DSM, the reactive relationships reduce to a proper level, and the relative relationship among the actions will also be kept unalterable. The basic approaches reconstructing DSM are as follows[5].

Switching rows. If the elements in the ith row are all 0 , denoted as $a_{i j}=0(j=1,2, \cdots, m)$, we can affirm that the action of $A_{i}$ cannot receive any information form the other actions. Thus, the action of $A_{i}$ should be placed in the foremost position, which shows that the action of $A_{i}$ should be executed earlier than the other actions. At the same time, the corresponding elements in the row and line that the action of $A_{i}$ is placed should be deleted. We should not stop switching all the rows until all the actions are analyzed.

Switching lines. If the elements in the jth line are all 0 , denoted as $a_{i j}=0(i=1,2, \cdots, m)$, we can affirm that the action of ${ }^{A_{i}}$ cannot output any information to the other actions. Thus, the action of $A_{i}$ should be placed in the last position, 
which shows that the action of $A_{i}$ should be executed later than the other actions. At the same time, the corresponding elements in the row and line that the action of $A_{i}$ is placed should be deleted. We should not stop switching all the rows until all the actions are analyzed.

Repeating the above two approaches. We should not stop repeating the above two approaches until the DSM becomes a matrix that all the elements lying on the diagonal are denoted as 0 . Thus, the reactive relationships among the actions will become fewer, and the efficiency of parallel processing will be higher.

\section{IMPLEMENTATION OF THE METHOD}

Generally, we can build various flow of ERD with the diffident goals. For example, the flow of ERD for the equipment type can be organized as the figure 3. In this flow, the six steps are studied, including equipment operation conception design, equipment capabilities requirement analysis, equipment function requirement analysis, equipment structure requirement analysis, equipment typical operation tasks validation and equipment typical operation nodes analysis. The final goal of this flow is to propose the development requirement of the equipment type, including the function requirement, the structure requirement and the performance parameter requirement.

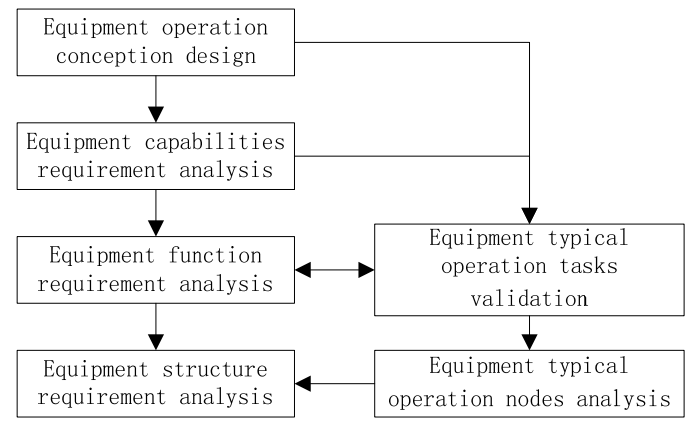

FIGURE III. THE FLOW OF ERD FOR EQUIPMENT TYPE.

\section{A. Constructing the DSM}

Assume that the six actions are denoted as $A_{1}, A_{2}, A_{3}, A_{4}, A_{5}$ and $A_{6}$. According to the definition of the DSM, the DSM describing the flow of ERD for equipment type can be defined as the initial DSM in the figure 4 . In this DSM, the elements denoted as 1 show that there are information stream between the corresponding two actions. Contrarily, the elements denoted as 0 show that there in no information stream between the corresponding two actions.

\section{B. Reconstructing the DSM}

According to the approaches reconstructing the DSM, the action of $A_{1}$ receives nothing from the other actions, so it is placed in the foremost position in the matrix as the initial. The action of $A_{2}$ also receive nothing from the actions of
$A_{3}, A_{4}, A_{5}$ and $A_{6}$, so it is placed in the foremost position in the matrix constructed by the actions of $A_{2}, A_{3}, A_{4}, A_{5}$ and $A_{6}$. And the action of $A_{4}$ output nothing to the actions of $A_{3}, A_{5}$ and $A_{6}$, so it is placed in the last position in the matrix constructed by the actions of $A_{3}, A_{4}, A_{5}$ and $A_{6}$. Thus, the reconstructed DSM is showed as figure 4 .

\begin{tabular}{|c|c|c|c|c|c|c|c|c|c|c|c|c|}
\hline$A_{1}$ & $A_{2}$ & $A_{3}$ & $A_{4}$ & $A_{5}$ & $A_{6}$ & & $A_{1}$ & $A_{2}$ & $A_{3}$ & $A_{5}$ & $A_{6}$ & $A_{4}$ \\
\hline$\left[A_{1}\right.$ & 0 & 0 & 0 & 0 & 0 & $A_{1}$ & $A_{1}$ & 0 & 0 & 0 & 0 & 0 \\
\hline 1 & $A_{2}$ & 0 & 0 & 0 & 0 & $A_{2}$ & 1 & $A_{2}$ & 0 & ) & 0 & 0 \\
\hline 0 & 1 & $A_{3}$ & 0 & 1 & 0 & $A_{3}$ & 0 & 1 & $A_{3}$ & 1 & 0 & 0 \\
\hline 0 & 0 & 1 & $A_{4}$ & 0 & 1 & $A_{5}$ & 1 & 1 & 1 & $4_{5}$ & 0 & 0 \\
\hline 1 & 1 & 1 & 0 & $A_{5}$ & 0 & $A_{6}$ & 0 & 0 & 0 & 1 & $A_{6}$ & 0 \\
\hline 0 & 0 & 0 & 0 & 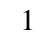 & $t_{16}$ & $A_{4}$ & 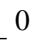 & 0 & 1 & 0 & 1 & $A_{4}$ \\
\hline
\end{tabular}

(a)the initial DSM $\quad$ (b) the reconstructed DSM

FIGURE IV. DSM DESCRIBING THE FLOW OF ERD FOR EQUIPMENT TYPE.

Contrasting the initial DSM and the reconstructed DSM, the number of the reactive relationships reduces to 1 , which shows that the parallel works are organized easier.

\section{Reconstructing the flow}

According to the reconstructed DSM showed in figure 5, the executed order of the actions follows the arranged order of the actions in the DSM. The elements denoted as 1 show that there are information streams between the corresponding two actions. So the reconstructed flow ERD for equipment type can be described as the figure 6 .

In the reconstructed flow, the six actions is divided into three groups. Every group includes some actions. And the relationships among the groups are series-wound. While the reactive relationship only exits inside the group 2. Thus, collaborative works among groups become more simple, and the efficiency of ERD will be higher[6].

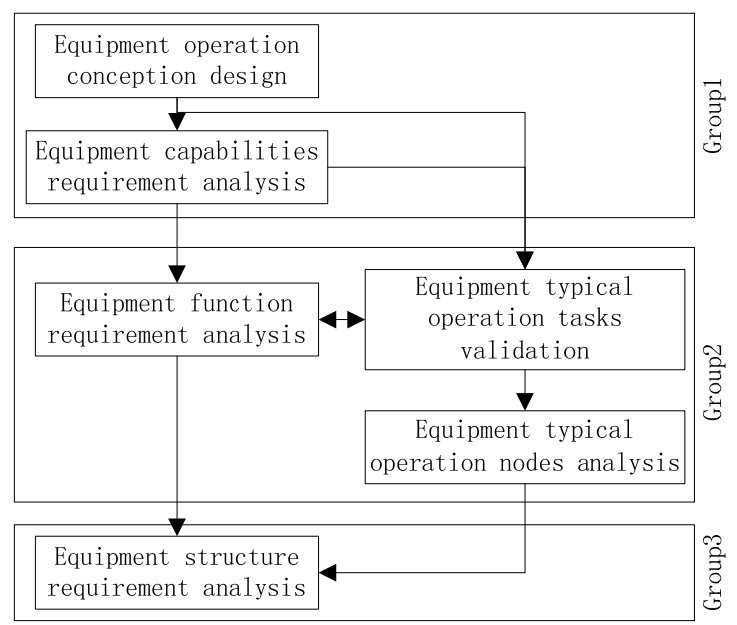

FIGURE V. THE OPTIMIZED FLOW OF ERD FOR EQUIPMENT TYPE. 


\section{SUMMARY}

It is an effective method to optimize the collaborative flow of ERD. This method will help to simply the interaction among the actions, and shorten the work time. However, with the increasing dimension of the DSM, the method reconstructing DSM will be not useful. In the future, the new method reconstructing DSM must be proposed. And the method proposed in this paper will be more perfect.

\section{REFERENCES}

[1] Y. P. Fan, Q. S. Guo, G. Mu, et al, Complexity of weapon system of systems requirement development and its solution. Systems Engineering and Electronics, 36(7),pp.1320-1327, 2014.

[2] Q. L. Li, Q. S. Guo, Research on the basic questions about the demonstration of equipment requirement based on capability. Journal of the Academy of Equipment command \&Technology, 20(5),pp.24-27, 2009.

[3] Q. S. Guo, K. Wang, Y. P. Fan, et al, Requirement analysis method for weapon equipment. Journal of Academy of Armored Force Engineering, 27(5),pp.8-12, 2013.

[4] L. N. Xu, H. M. Zhang, Y. K. Zhang, Research on process reconfiguration with DSM in collaborative design.China Engineering Science, 8(5),pp.52-57, 2006.

[5] W. D. Huang, Research on management of multi-model collaborative products design and its Key Technologies, Nanjing: Nanjing University of Aeronautics and Astronautics,pp.37-46,2009.

[6] L. N. Xu, H. M. Zhang, Y. K. Zhang, Multidisciplinary cooperative design with DSM(Design Structure Matrix).China Mechanical Engineering, 16(12),pp.1035-1038,2005.. 